\title{
POTÊNCIA ATADA NA MÁXIMA FASE ESTÁVEL DE LACTATO E ÍNDICES DO DESEMPENHO AERÓBIO DE NADO
}

\author{
TETHER-POWER AT MAXIMAL LACTATE STEADY-STATE AND ENDURANCE INDEXES OF \\ SWIMMING PERFORMANCE
}

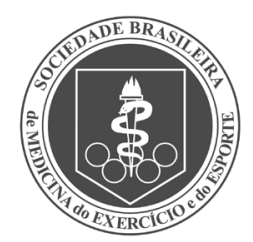

Artigo Original

\author{
POTENCIA ATADA EN LA MAXIMA FASE ESTABLE DEL LACTATO Y RENDIMIENTO \\ AERÓBICO DE NADO
}

\author{
Dalton Müller Pessôa Filho' \\ (Educador Físico) \\ Camila Coelho Greco² \\ (Educador Físico) \\ Benedito Sérgio Denadai \\ (Educador Físico) \\ 1. Universidade Estadual Paulista, \\ Bauru, SP, Brasil. \\ 2. Universidade Estadual Paulista, \\ Rio Claro, SP, Brasil.
}

\section{Correspondência:}

Benedito Sérgio Denadai

Laboratório de Avaliação da

Performance Huamana, Instituto

de Biociência, UNESP. Av 24A, 1515,

Bela Vista, Rio Claro, SP, 13.506-900

bdenadai@rc.unespbr

\begin{abstract}
RESUMO
Introdução: A perspectiva do nado atado constituir um contexto válido para a avaliação aeróbia de nadadores foi investigada no presente estudo. Objetivo: Analisar a relação entre a potência em máxima fase estável de lactato no nado atado $\left(P_{\text {AtadaMFel }}\right)$ com seu respectivo índice em nado desimpedido (velocidade em MFEL, $v_{\text {MFEL }}$ ) e com outros índices da aptidão aeróbia e desempenho de nado crawl. Métodos: Dez nadadores (16,6 $\pm 1,4$ anos) foram submetidos às estimativas de: (a) $P_{\text {Atadocrítica }}$ (transformação da assíntota do modelo

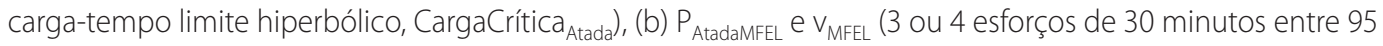
a 105\% da Carga Crítica $a_{\text {Atada }}$ e entre 85 a 95\% da velocidade máxima nos 400 m, respectivamente), (c) teste progressivo (79-100\% da $\mathrm{V}_{400 \mathrm{~m}}$, com incrementos de $3 \%$ ) para a determinação da velocidade no ponto de inflexão $(v P I)$, e (d) testes de desempenho nas distâncias de $400\left(v_{400 m}\right), 800\left(v_{800 m}\right)$ e $1500\left(v_{1500 m}\right)$ metros. Os coeficientes de Pearson e de variância analisaram as correlações entre os parâmetros aeróbios e destes com o desempenho. $O$ teste de Bland-Altman foi utilizado para analisar a concordância entre as concentrações de lactato nas avaliações aeróbias. Resultados: $O$ valor de $P_{\text {AtadamfEL }}(89,2 \pm 15,1 \mathrm{~W}$ ) apresentou potencial similar de explicação da variância nos desempenhos em $v_{400 \mathrm{~m}}\left(1,29 \pm 0,11 \mathrm{~m} \cdot \mathrm{s}^{-1}, R^{2}=0,700\right), v_{800 \mathrm{~m}}\left(1,23 \pm 0,12 \mathrm{~m} \cdot \mathrm{s}^{-1}\right.$, $\left.R^{2}=0,770\right)$ e $v_{1500 m}\left(1,21 \pm 0,12 \mathrm{~m} \cdot \mathrm{s}^{-1}, R^{2}=0,698\right)$ tal como $v_{\text {MFEL }}\left(1,17 \pm 0,11 \mathrm{~m} \cdot \mathrm{s}^{-1}\right)$ e vPI $\left(1,19 \pm 0,11 \mathrm{~m} \cdot \mathrm{s}^{-1}\right)$. As concentrações de lactato sanguíneo em $P_{\text {AtadamFEL }}, V_{\text {MFEL }}$ e vPI não diferem entre si e distribuíram-se dentro dos limites de concordância. Conclusão: Pode-se concluir que a aplicação de MFEL em nado atado mostrou-se válida e promissora para a avaliação aeróbia de nadadores.
\end{abstract}

Palavras-chave: aptidão física, resistência física, provas de rendimento, ácido láctico, natação.

\section{ABSTRACT}

Introduction: The validity of tethered-swimming to assess swimmer endurance capacity was analyzed in the present research. Objective: The aim of this study was to analyse the relationship between the mechanical power at maximal lactate steady state ( $P_{\text {TethmLSS }}$ ) with its respective parameter in free swimming (velocity at MLSS, $V_{\text {MLSS }}$ ) and with others indices of aerobic evaluation and endurance performance in free crawl swimming. Methods: Ten male swimmers (16.6 \pm 1.4 years old) were submitted to the following measurements: (a) $C P_{T e t h}$ (estimated from non-linear asymptote for load and time-limited plotting, Critical Load, (b) $P_{\text {TethMLSS }}$ and $v_{\text {MLSS }}$ ( 3 or 4 trials ranging from 95 to 105\% of Critical Load and 85 to $95 \%$ of 400-m crawl performance, respectively), (c) an incremental protocol (79-100\% of $v_{400 m}$ with step increments of 3\%) to assess velocity at inflexion point (VIP) and, (d) swimming performance of $400\left(\mathrm{~V}_{400 \mathrm{~m}}\right), 800\left(\mathrm{~V}_{800 \mathrm{~m}}\right)$ and $1500\left(v_{1500 m}\right)$ meters. Pearson and variance coefficients were used to analyze the relationships between aerobic parameters and performance indexes. Bland-Altman test was used to analyze the agreement between blood lactate values. Significance was set at $p \leq 0.05$ for all analysis. Results: Values of $P_{\text {Tethm L.sS }}(89.2 \pm 15.1 \mathrm{~W})$ presented similar potential to explain the variance of performance in $v_{400 \mathrm{~m}}\left(1.29 \pm 0.11 \mathrm{~m} \cdot \mathrm{s}^{-1}, R^{2}=0.700\right), v_{800 \mathrm{~m}}\left(1.23 \pm 0.12 \mathrm{~m} \cdot \mathrm{s}^{-1}, R^{2}=0.770\right)$ and $v_{1500 \mathrm{~m}}$ $\left(1.21 \pm 0.12 \mathrm{~m} \cdot \mathrm{s}^{-1}, R^{2}=0.698\right)$, as well as $v_{\text {MLSS }}\left(1.17 \pm 0.11 \mathrm{~m} \cdot \mathrm{s}^{-1}\right)$ and $v I P\left(1.19 \pm 0.11 \mathrm{~m} \cdot \mathrm{s}^{-1}\right)$. Blood lactate levels at $P_{\text {TethmLSS }}$ $V_{\text {MLSS }}$ and VIP were not different and were distributed within the concordance limits. Conclusion: We concluded that MFEL in tethered-swimming is a valid and promising protocol for aerobic evaluation of swimmers.

Keywords: physical fitness, physical endurance, performance tests, lactic, acid, swimming.

\section{RESUMEN}

Introducción: La perspectiva del nado atado en constituir un contexto válido para la evaluación aeróbica de nadadores fue investigada en el presente estudio. Objetivo: Analizar la relación entre la potencia en máxima fase estable de lactato en el nado atado $\left(P_{\text {AtadamEEL }}\right)$ con su respectivo índice en nado libre (velocidad en MFEL, $\left.V_{M F E L}\right)$, y con otros índices de aptitud aeróbica en nado crawl. Métodos: Diez nadadores (16,6 1,4 años) fueron sometidos a la estimativa de (a) $P_{\text {Atadacritica }}$ (transformación de la asíntota del modelo carga-tiempo límite hiperbólico, Car-

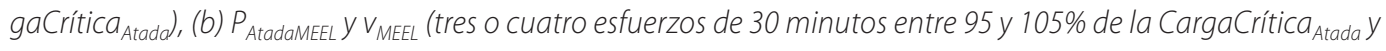


entre 85-95\% de la velocidad máxima en los 400 metros, respectivamente), (c) prueba progresiva (79-100\% de la $V_{400 m}$ con incrementos de 3\%) para la determinación de la velocidad en el punto de inflexión (vPl), y (d) pruebas de rendimiento en las distancias de $400\left(V_{400 \mathrm{~m}}\right), 800\left(\mathrm{~V}_{800 \mathrm{~m}}\right)$ y $1500\left(\mathrm{~V}_{1500} \mathrm{~m}\right)$ metros. Los coeficientes de Pearson y de variancia analizaron las correlaciones entre los parámetros aeróbicos y de éstos con el desempeño. El test de Bland-Altman fue utilizado para analizar la concordancia entre las concentraciones de lactato en las evaluacio-

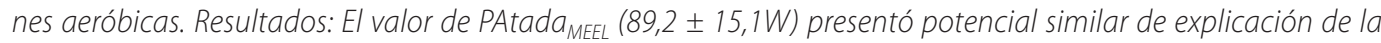
variancia en los desempeños en $v_{400 m}\left(1,29 \pm 0,11 \mathrm{~m} \cdot \mathrm{s}^{-1}, R^{2}=0,700\right), v_{800} m\left(1,23 \pm 0,12 \mathrm{~m} \cdot \mathrm{s}^{-1}, R^{2}=0.770\right)$ y $v_{1500 \mathrm{~m}}$ $\left(1,21 \pm 0,12 \mathrm{~m} \cdot \mathrm{s}^{-1}, R^{2}=0,698\right)$ tal como vMFEL $\left(1,17 \pm 0,11 \mathrm{~m} \cdot \mathrm{s}^{-1}\right)$ y $v^{P I}(1,19 \pm 0,11 \mathrm{~m} \cdot \mathrm{s}-1)$. Las concentraciones de lactato sanguíneo en la $P A_{\text {tadaMEEL, }} V_{\text {MEEL }}$ y VIP no se diferenciaron entre sí y se distribuyeron dentro de los límites de concordancia. Conclusión: Se puede concluir que la aplicación de MFEL en nado atado se mostró válida y promisora para la evaluación aeróbica de nadadores.

Palabras clave: aptitud física, resistencia física, pruebas de rendimiento, ácido láctico, natación.

\section{INTRODUÇÃO}

Em termos qualitativos, a máxima fase estável da concentração do lactato sanguíneo (MFEL) discrimina a intensidade do exercício contínuo que é limitada pelas reservas de energia daquelas cujo término decorre da perturbação da homeostase celular ${ }^{1}$. Ao se exercitar na MFEL, a exaustão ocorrerá provavelmente pela inabilidade em fornecer energia, via glicogenólise, suficiente para atender a demanda contrátil dos músculos, permitindo por isso avaliar a limitação das reservas de glicogênio². Além do tamanho da reserva de glicogênio, outros determinantes da modulação da concentração de lactato em MFEL são: (a) a taxa de ativação da glicólise, (b) volume de distribuição do lactato pelos compartimentos de água no corpo, (c) mecanismos de transporte ativo/passivo do lactato pelos compartimentos, (d) disponibilidade de oxigênio nos tecidos, (e) nível de treinamento e ( $f$ ) massa muscular primariamente engajada na atividade ${ }^{2,3}$. A melhora nos processos de transporte dentro e entre as células com treinamento aeróbio seria o fator preponderante no aumento da intensidade de esforço, ou concentração de lactato, relacionada à MFEL, constituindo-a em uma referência padrão para a determinação da capacidade aeróbia ${ }^{3}$.

Devido à grande variabilidade da concentração de lactato em $\operatorname{MFEL}\left(2,2-6,7\right.$ mmol.. $\left.{ }^{-1}\right)$ e ao extenso protocolo de determinação ${ }^{2,3}$, a intensidade correspondente à MFEL (velocidade ou carga) tem sido relacionada aos demais índices representativos de limiares de respostas fisiológicas obtidos em testes incrementais, como as velocidades respectivas: (a) ao início do acúmulo do lactato no sangue (v-OBLA), (b) ao ponto de incremento do lactato acima dos valores de repouso (LL); (c) ao incremento do equivalente ventilatório para o $\mathrm{VO}_{2}\left(\mathrm{~V}_{\mathrm{E}} \mathrm{NO}_{2}\right)\left(\mathrm{VT}_{1}\right)$; (d) ao incremento do equivalente ventilatório para o $\mathrm{VCO}_{2}\left(\mathrm{~V}_{\mathrm{E}} \mathrm{NCO}_{2}\right)\left(\mathrm{VT}_{2}\right)$; e à velocidade crítica (VC, assíntota da relação velocidade-tempo $)^{2,4-6}$.

Para a obtenção dos índices MFEL e VC e suas respectivas respostas fisiológicas durante a natação, a manipulação da intensidade do exercício tem sido realizada pelo controle da velocidade e dos parâmetros gestuais da braçada (distância por braçada - Db, em metros por braçada e frequência de braçada - Fb, em ciclos por minuto). O uso de controladores da velocidade por estímulos visuais e sonoros favorecem a manutenção de uma velocidade e ritmo gestual constante, sobretudo em estágios com intensidade muito baixa ou alta de protocolos retangulares ou progressivos. Estes procedimentos diferem daqueles habitualmente empregados no treinamento e, consequentemente, demandam recursos externos para atender as premissas de uniformidade (ritmo e/ou resposta metabólica) durante os estágios de uma avaliação pelo perfil lactacidêmico ${ }^{7}$. Na natação, essa preocupação é ainda maior devido à existência de uma oscilação da velocidade linear de nado em cada ciclo de braçada (variação intra-ciclo da velocidade), que aumenta com o incremento da velocidade e pode comprometer a mecânica gestual da braçadå .
As avaliações empregando testes de intensidade constante na natação mostram que a MFEL é coincidente com o ponto de pico de Db, sugerindo sua aplicação não somente para a avaliação e desenvolvimento da aptidão para desempenhos de longa duração ${ }^{9}$, mas também para controle e aprimoramento da técnica de nado ${ }^{4}$. Por sua vez, a VC também mostra-se associada aos índices da capacidade aeróbia, como a velocidade do teste de 30 minutos, à velocidade média nos $400 \mathrm{~m}$, ao LL e OBLA (ponto referente à concentração de 4 mmol.L-1 de lactato no sangue), apesar de apresentar-se em valores diferentes em relação à estes índices ${ }^{10}$.

Para Kjendlie e Thorsvald ${ }^{11}$, o nado atado (sem deslocamento, o que difere do semi-atado) é um método confiável para estimar a força atada de nado, uma vez que medidas de teste e re-teste produziram elevados coeficientes de correlação e baixos coeficientes de variação para desempenhos realizados em dias e períodos diferentes. Esses autores também consideram o nado atado um contexto em que a aplicação de força oscila pouco, pois há uma melhor distribuição da força e velocidade do hidrofólio durante o ciclo da braçada. Entretanto, para o nosso conhecimento, não há estudos que tenham analisado a validade da utilização do nado atado na avaliação de índices aeróbios pela relação com índices aeróbios lactacidêmicos (MFEL, limiares anaeróbios) e de performance (velocidade em 400, 800 e 1500 metros) em nado desimpedido (não atado).

Com base nestas informações, este estudo analisou a importância do nado atado como instrumento de avaliação aeróbia e predição do rendimento em provas de média a longa duração, considerando o fato que essas provas (200 a 1500 m) apresentam, conforme Dekerle et al. ${ }^{12}$, uma elevada demanda oxidativa (durações entre $\sim 1$ min $50 \mathrm{~s} \mathrm{a}$ 15 min). Para tanto, é proposto o delineamento de protocolos com intensidade constante baseados no gerenciamento de cargas externas para: (1) determinar a potência correspondente à carga em MFEL $\left(P_{\text {AtadaMfel }}\right) ;(2)$ associar a $P_{\text {Atadamfel }}$ aos índices de desempenhos em provas de média e longa duração em nado livre (400, 800 e 1500 m); e (3) comparar a magnitude dessas associações com aquelas apresentadas por índices aeróbios lactacidêmicos e de desempenho obtidos por avaliações aeróbias em nado livre ( $\mathrm{v}_{\text {MFEL }}$ e limiar anaeróbio por cinética lacidêmica).

\section{MATERIAL E MÉTODOS}

\section{Sujeitos}

Foram analisados 10 nadadores do sexo masculino (16,6 $\pm 1,4$ anos, $69,8 \pm 9,5 \mathrm{~kg}, 175,8 \pm 4,6 \mathrm{~cm}$ e 7,0 \pm 4,0\% de gordura corporal), regularmente engajados em treinamento para natação e familiarizados com o nado atado. No período em que o estudo foi realizado, a equipe de 
nadadores ocupava a 9a colocação na classificação regional do Estado de São Paulo. Todos os nadadores assinaram um termo de consentimento livre e espontâneo para participação. Esta pesquisa foi submetida e aprovada pelo Comitê de Ética em Pesquisa (CEP) da FC - UNESP/ Bauru, sob o processo CAEE: 02402512.7.0000.5398.

\section{Delineamento Experimental}

Avaliações antropométricas e de velocidade máxima de nado crawl modelaram a carga máxima a ser empregada nos testes de exaustão e de intensidade constante. Essa referência de carga foi fracionada e o tempo de exaustão de nado crawl-atado foi registrado para a constru-

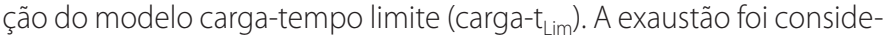
rada o momento em que o nadador não se mostrou capaz de manter a posição e, então, foi tracionado para trás pelo sistema, ou simplesmente desistiu. Após os testes de exaustão em nado atado, deu-se início aos testes de desempenho em nado desimpedido (crawl) nas distâncias de 400, 800 e 1500 metros. Os esforços foram executados em intervalos de 24 horas, durante as sessões de treinamento e após a fase inicial (aquecimento) da sessão de treinamento. A determinação da MFEL foi realizada por quatro testes constantes com duração de 30 minutos, em cada condição de nado (atado e desimpedido). A referência inicial foi a velocidade no desempenho em 400 metros para o nado desimpedido e a CargaCrítica $a_{\text {Atada }}$ (assíntota carga-t Lim $_{\text {Li }}$ por ajuste hiperbólico de dois parâmetros) para o nado atado. Todos os testes foram completados em até três semanas para evitar efeitos decorrentes do treinamento sobre o desempenho nos testes (subestimativa da intensidade de esforço nos testes preditivos por defasagem da referência inicial de carga máxima e desempenhos nos $400 \mathrm{~m}$ ). Foi priorizada a aplicação dos testes no período preparatório básico do treinamento, não concorrendo com o tipo de estímulo empregado na unidade de treinamento. Todos os testes foram realizados no nado crawl, em piscina de $50 \mathrm{~m}$, não coberta, com temperatura da água entre 26 a $28^{\circ} \mathrm{C}$, sempre no mesmo período do dia (vespertino), entre os meses de janeiro e fevereiro (verão). O volume da sessão diária de treinamento, no período da intervenção, era de $5.000 \mathrm{~m}$, sendo $70 \%$ executado com intensidade moderada a pesada, em médias a longas distâncias.

\section{Teste com cargas constantes e determinação da relação i- $\mathbf{t}_{\text {Lim }}$}

O protocolo deste modelo previu esforços contínuos, com intensidade de nado graduada por cargas constantes, que conduzissem à exaustão voluntária em 3 a 15 minutos, conforme previsto em outras modalidades esportivas ${ }^{3}$ e na natação ${ }^{13}$. A variável mecânica CargaCrítica $_{\text {Atada }}$ foi determinada seguindo as recomendações de Pessôa Filho et al. ${ }^{14}$ e Pessôa Filho e Denadai ${ }^{15}$. Para estes autores, primeiramente obtém-se um índice da força máxima em nado desimpedido. Esse índice pode ser estimado baseando-se nas relações entre força de arrasto ativo (Fr) e proporcionalidade corporal (A), conforme Toussaint et al. ${ }^{16}$, pela equação:

\section{$F r=A \times v^{2}$}

onde $A$ é a constante de proporcionalidade, estimada em cada sujeito, a partir do algoritmo [(0,35 x massa corporal) +2$]$, sendo a massa corporal dos sujeitos em $\mathrm{kg}$ e a velocidade registrada em $\mathrm{m}^{-1} \mathrm{~s}^{-1}$. O protocolo consistiu em registrar o tempo de nado crawl, em máxima velocidade possível, por uma distância de $15 \mathrm{~m}$, que foi demarcado entre 10 e $25 \mathrm{~m}$ de uma piscina de $50 \mathrm{~m}$ para evitar a influência do tempo de reação, impulsão no bloco de partida, deslizamento na fase submersa, aceleração/desaceleração. O resultado refere-se à carga $(\mathrm{N})$, que foi fracionada em uma margem de intensidades (75-105\%) e anexada ao sistema de polias de base móvel (dispositivo de nado atado). Este sistema é composto por cordas paralelas, com cinco roldanas fixas no ápice e quatro móveis na base, formando a talha exponencial. A força de tração ao final do sistema coincidiu com a fração da intensidade da carga aplicada. O sistema é acoplado ao nadador por um cabo de aço inelástico, preso a um cinto posicionado na cintura pélvica.

A partir do tempo de exaustão no primeiro teste a 100\% da carga estimada pela Eq. 1, os testes seguintes foram ajustados em $\pm 5 \%$ para a obtenção de três esforços com exaustão entre 3-15 minutos, conforme recomendação de Bishop et al. ${ }^{17}$. A relação entre intensidade da carga e duração dos esforços compôs o modelo carga- $t_{\text {Lim }}$ pela equação:

$$
t=\frac{\text { CTA }}{C-\text { CargaCrítica }}
$$

onde CTA (kg.m.s. ${ }^{-1}$ é a carga de trabalho anaeróbio; e CargaCrítica é a assíntota do modelo em Newtons (CargaCrítica ${ }_{\text {Atada }}$ ). Esse modelo hiperbólico de 2-parâmetros (Eq. 2) tem sido comumente aplicado na composição do modelo de velocidade crítica (VC) para o nado livre ${ }^{13,18,19}$. No nado atado, esse modelo foi adaptado do modelo impulso-tempo limite (i-t $t_{\text {Lim }}$ ) de Ikuta et al. ${ }^{20}$, conforme demonstrado por Pessôa Filho e Denadai' ${ }^{15}$.

A variável mecânica potência mecânica atada $\left(P_{\text {Atado }}\right)$ foi derivada dos parâmetros de carga da Eq. 2 e quantificada pela equação.

$$
P_{\text {Atado }}(W)=\operatorname{Carga}(N) \times v_{\text {Hidrof }}
$$

onde $v_{\text {Hidrof }}$ Corresponde à velocidade do hidrofólio, que em condições de nado atado, pode ser estimada, segundo Pessôa Filho et al. ${ }^{14}$ e Pessôa Filho e Denadai ${ }^{21}$, por:

$$
v_{\text {Hidrof }}=\left(\operatorname{Carga}(N) /\left(0,5 C x_{\text {Hidrof }} \times S_{\text {Hidrof }} \times p\right)\right)^{1 / 2}
$$

onde $\mathrm{C}_{\text {Hidrof }}$ é o coeficiente de arrasto do hidrofólio, estimado em 2,2 e proposto como uma constante ao longo das diferentes intensidades de nado ${ }^{22 ;}$; $\rho^{\prime \prime}$ é a densidade da água, considerada em $1000 \mathrm{~kg} \cdot \mathrm{m}^{-3}$; $\mathrm{S}_{\text {Hidrof }}$ a área de seção transversa do hidrofólio do nadador exposta ao fluído, determinada pelo volume do hidrofólio elevado a dois terços $\left(V_{\text {Hidrof }}{ }^{2 / 3}\right) . O V_{\text {Hidrof }}{ }^{2 / 3}$ foi determinado pela massa e densidade da mão e do antebraço, obtidos pela massa corporal de cada sujeito, empregando o modelo antropométrico de Hatz.

\section{Determinação da MFEL no crawl atado e desimpedido:}

Os nadadores realizaram quatro esforços de 30 minutos, em nado crawl-atado, com cargas relativas a 95, 97,5, 100 e 102,5\% da carga referente à $\mathrm{P}_{\text {Atadacritica }}{ }^{21}$. No nado crawl desimpedido, a velocidade nos quatros testes de 30 minutos esteve entre $85,5 \%$ a $95,0 \%$ do desempenho máximo em $400 \mathrm{~m}\left(\mathrm{~V}_{400 \mathrm{~m}}\right)$, com incrementos de 2,5\% na velocidade, conforme sugerido por Dekerle et al. ${ }^{10}$.

Em ambos os testes, as intensidades foram realizadas de forma aleatória e os esforços interrompidos duas vezes, ao $10^{\circ}$ e $30^{\circ}$ minutos para a coleta de sangue. A MFEL foi considerada a maior intensidade (potência ou velocidade de nado) em que a concentração de lactato não se mostrou superior a $1 \mathrm{mmol}^{-L^{-1}}$ entre os $10^{\circ}$ e $30^{\circ}$ minutos do esforço ${ }^{2}$, sendo determinadas a $P_{\text {AtadamFEL }}$ e $v_{\text {MFEL }}$. Contudo, caso a MFEL não ocorresse dentro das faixas de intensidade previstas, incrementos na intensidade foram efetuados na taxa de 2,5\%. Quando a exaustão ocorreu antes do $30^{\circ}$ minuto, a intensidade imediatamente anterior foi considerada, para ambos os protocolos, como MFEL.

\section{Determinação do ponto de inflexão no nado crawl desimpedido}

O ponto de inflexão foi determinado pelas relações entre a velocidade e a concentração de lactato, em um teste incremental contínuo (300 m por estágio) até a exaustão. Os sujeitos foram submetidos aos esforços em intensidade correspondente a 79 - 100\% da velocidade 
respectiva ao desempenho máximo em 400 m no crawl desimpedido $\left(v_{400 m}\right)$, com duração em torno de 3 minutos, incrementos de $3 \%$ e intervalos de pausa de 30 s para que coletas de sangue fossem efetuadas e o Ponto de Inflexão (PI) fosse identificado, a partir do procedimento delineado por Beaver et al. ${ }^{23}$ e revisado por Bentley et al. ${ }^{24}$. Este consiste em traçar as coordenadas lactato vs. velocidade, no plano cartesiano, e determinar a velocidade correspondente à intersecção de duas retas $(F(y)=a+b x)$ para o conjunto de coordenadas (bi-segmentação), fornecendo o ponto de inflexão para a velocidade de nado (vPI). A segmentação dos dados foi realizada pela inspeção visual, discriminando o aumento sustentado da concentração sangüínea de lactato acima dos valores basais do incremento abrupto em direção aos valores máximos. Em todos os testes, as amostragens sangüíneas foram obtidas por amostras de $25 \mu$ lde sangue arterializado do lóbulo da orelha, por meio capilar heparinizado e previamente calibrado. As amostras foram armazenadas em tubos de $5 \mathrm{ml}$ calibrados com $50 \mu \mathrm{l}$ de $\mathrm{NaF}$ a $1 \%$ e congelados, quando não imediatamente analisados em um analisador eletroquímico (YSI 2300, Yellow Springs ${ }^{\circledR}$, Ohio, USA).

\section{Testes de desempenho em médias e longas distâncias de nado desimpedido}

A velocidade média nas distâncias de 400, 800 e $1500 \mathrm{~m}\left(\mathrm{v}_{400 \mathrm{~m}}, \mathrm{~V}_{800 \mathrm{~m}}\right.$ e $v_{1500 \mathrm{~m}}$ ) foi determinada durante as sessões de treinamento. Após aquecimento inicial, dentro e fora da água, os nadadores foram instruídos a desempenhar esforços nas distâncias previstas, em velocidade máxima para o percurso e de forma contínua. O tempo para cada distância foi registrado por cronômetro manual, sendo adotado o melhor dentre dois desempenhos de cada distância. Cada desempenho foi realizado com intervalo de 24 horas, como unidade de treinamento.

\section{Análise estatística}

As variáveis $P_{\text {AtadaMFEL, }}, V_{\text {MFEL }}$ e vPI foram testadas quanto à normalidade de suas distribuições pelo teste de Shapiro-Wilk. Adotou-se: (a) o teste ANOVA (uma entrada, conjugado à Scheffé como post-hoc) para averiguar as diferenças entre as concentrações sanguíneas de lactato em $P_{\text {AtadamFel, }} v_{\text {MFEL }}$ e vPI. Assim como o teste Bland-Altman analisou a concordâncias entre essas concentrações de lactato; (b) o coeficiente de Pearson ( $r$ ) analisou o tipo e magnitude das correlações entre os parâmetros ( $P_{\text {AtadaMfEL }} V_{\text {MFEL }}$ e vPI) e destes com as distâncias de desempenho $\left(V_{400 m}, v_{800 m}\right.$ e $\left.v_{1500 m}\right)$; e (c) o potencial de explicação da variância nos desempenhos $\left(\mathrm{V}_{400 \mathrm{~m}}, \mathrm{~V}_{800 \mathrm{~m}}\right.$ e $\left.\mathrm{V}_{1500 \mathrm{~m}}\right)$ pelos parâmetros aeróbios $\left(P_{\text {AtadaMFEL }}, V_{\text {MFEL }}\right.$ e vPI) foi analisado pelo coeficiente de dispersão $\left(R^{2}\right)$, empregando o métodos dos quadrados mínimos. Em todas as análises foi adotado um nível de significância em $p \leq 0,05$. Todos os testes foram realizados no pacote estatístico SPSS 17.0.

\section{RESULTADOS}

A referência de carga máxima obtida pela Equação 1 foi 70,3 \pm 8,9 N, em velocidade de nado crawl para os $15 \mathrm{~m}$ de 1,63 $\pm 0,07 \mathrm{~m} \cdot \mathrm{s}^{-1}$, que foi equivalente à constante $26,4 \pm 3,3 \cdot v^{2}$. As avaliações de MFEL em nado desimpedido e atado estão detalhadas na tabela1. Todos os procedimentos de avaliação da capacidade aeróbia em nado atado e desimpedido são demonstrados na figura 1. As concentrações de lactato correspondentes à MFEL no nado crawl-atado (3,8 \pm 0,6 mmol. $\left.\mathrm{L}^{-1}\right)$ e no crawl desimpedido $\left(3,6 \pm 1,0 \mathrm{mmol}^{-L^{-1}}\right)$, bem como no ponto de inflexão $\left(3,4 \pm 1,1 \mathrm{mmol} . \mathrm{L}^{-1}\right)$ não apresentaram-se estatisticamente diferentes ( $\rho=0,856, \rho=0,563$ e $\rho=0,873$ ). As análises Bland \& Altman (figura 2) entre os perfis lactacidêmicos em MFEL no crawl-atado e desimpedido, e destes com a concentração de lactato no ponto de inflexão, apresentaram dispersões dentro dos limites de concordância ( $\pm 1,96$ DP),
Tabela 1. Parâmetros de lactacidemia, de carga/potência e desempenho nos modelos de máxima fase estável de lactato (MFEL) em nado crawl atado e desimpedido.

\begin{tabular}{|c|c|c|c|c|}
\hline Modelo & & \multicolumn{3}{|c|}{ Esforços } \\
\hline $\begin{array}{c}\text { MFEL: crawl } \\
\text { atado }\end{array}$ & & 10 & 20 & 30 \\
\hline & *\% Carga Crítica ${ }_{\text {Atada }}$ & $92,4 \pm 3,9$ & $95,4 \pm 4,6$ & $98,5 \pm 5,2$ \\
\hline & ${ }^{*}$ Lactato $_{\text {final }}\left(\mathrm{mmol} \mathrm{L}^{-1}\right)$ & $3,3 \pm 0,6$ & $4,5 \pm 1,5$ & $5,4 \pm 1,8$ \\
\hline & ${ }^{*} \Delta$ Lactato $\left(\mathrm{mmol}^{\circ} \mathrm{L}^{-1}\right)$ & $0,2 \pm 0,2$ & $0,6 \pm 0,6$ & $1,1 \pm 0,5$ \\
\hline & $P_{\text {AtadaMFEL }}(\mathrm{W})$ & \multicolumn{2}{|c|}{$89,2 \pm 15,1$} & \\
\hline & $\operatorname{Carga}_{\text {AtadaMFEL }}(\mathrm{N})$ & \multicolumn{2}{|c|}{$51,7 \pm 5,6$} & \\
\hline & \% Carga Crítica ${ }_{\text {Atada }}$ & \multicolumn{2}{|c|}{$96,2 \pm 6,2$} & \\
\hline & Lactato $_{\text {MFEL }}\left(\mathrm{mmol}^{\left.-\mathrm{L}^{-1}\right)}\right.$ & \multicolumn{2}{|c|}{$3,8 \pm 0,6$} & \\
\hline \multirow[t]{7}{*}{$\begin{array}{l}\text { MFEL: crawl } \\
\text { desimpedido }\end{array}$} & & & & \\
\hline & $\% \mathrm{~V}_{400}$ & $86,9 \pm 2,2$ & $90,3 \pm 1,8$ & $92,8 \pm 1,8$ \\
\hline & Lactato $_{\text {final }}\left(\mathrm{mmol} \mathrm{L}^{-1}\right)$ & $2,1 \pm 0,8$ & $3,3 \pm 1,1$ & $5,1 \pm 1,1$ \\
\hline & $\Delta$ Lactato $\left(\mathrm{mmol}^{\left.\mathrm{L} \mathrm{L}^{-1}\right)}\right.$ & $0,2 \pm 0,2$ & $0,5 \pm 0,3$ & $1,3 \pm 0,6$ \\
\hline & $v_{\text {MFEL }}\left(m \cdot s^{-1}\right)$ & \multicolumn{2}{|c|}{$1,17 \pm 0,11$} & \\
\hline & $\% \mathrm{~V}_{400}$ & \multicolumn{2}{|c|}{$90,7 \pm 2,2$} & \\
\hline & Lactato $_{\text {MFEL }}\left(\mathrm{mmol}^{-1} \mathrm{~L}^{-1}\right)$ & \multicolumn{2}{|c|}{$3,6 \pm 1,0$} & \\
\hline
\end{tabular}

*Obs: "\%Carga crítica "fração da força (N) correspondente à potência crítica no crawl atado determinada por ajuste hiperbólico de dois parâmetros; "Lactatofinal" concentração de lactato ao $30^{\circ}$ minuto; " $\triangle$ Lactato" variaçăo da concentração de lactato entre o 100 e o 300 minutos do esforço.
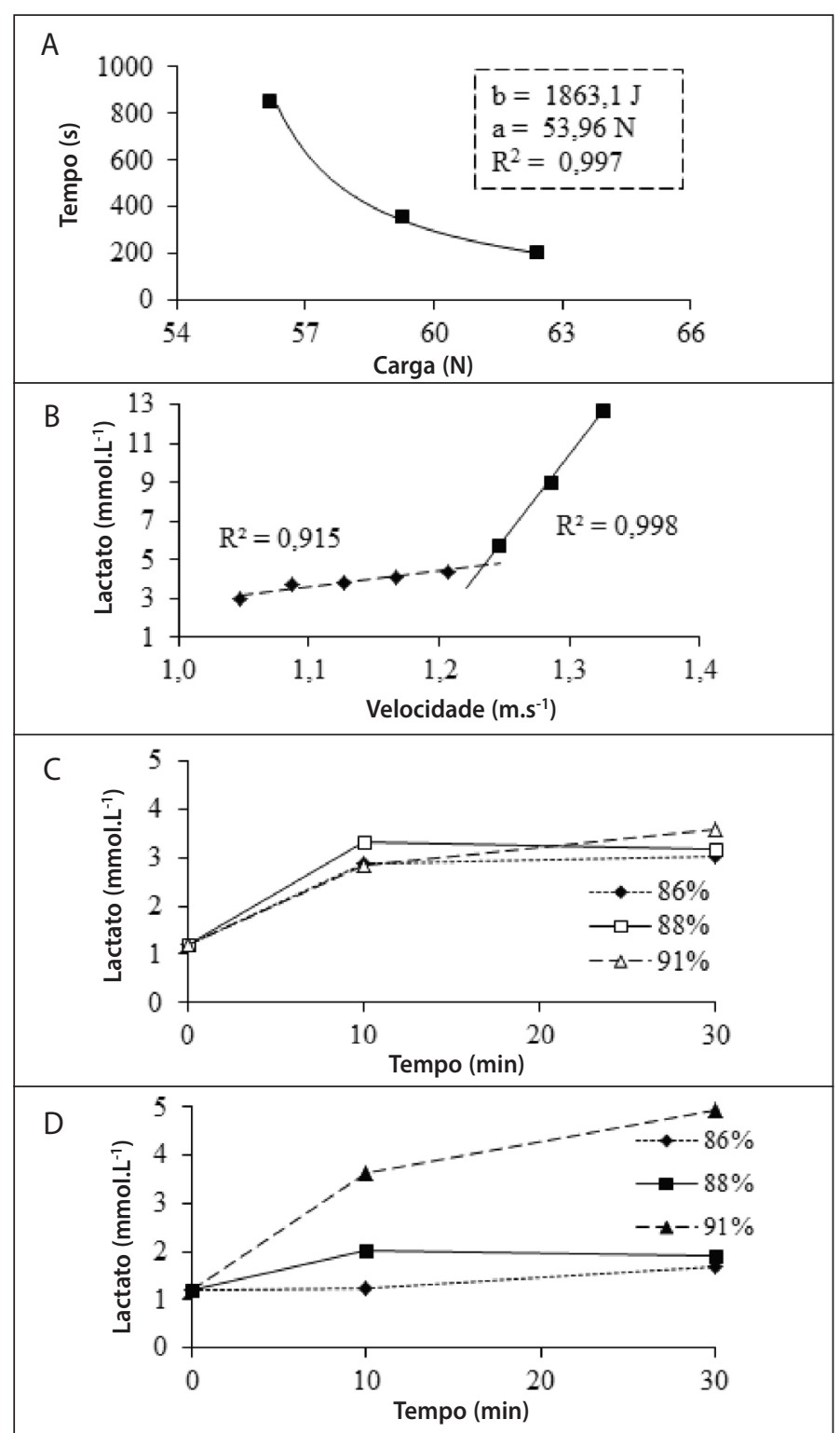

Figura 1. Métodos de avaliação aeróbia: modelo hiperbólico carga-t $t_{\text {Lim }}(A)$, em nado crawl atado ( $a=C T A ; b=$ CargaCrítica $_{\text {Atada }}$ ); cinética do lactato na determinação de vPI (B), em nado crawl desimpedido; determinação da máxima fase estável de lactato em teste constantes, por percentuais da CargaCrítica ${ }_{\text {Atada }}(C)$, e por percentuais da v400m. Sujeito 1. 
com tendência não significante, mas similares. O valor de potência correspondente à carga em MFEL não se mostrou diferente $(\rho=0,515$, T-teste independente) da potência $(94,6 \pm 21,6 \mathrm{~W})$ correspondente à CargaCítica ${ }_{\text {Atada }}(54,1 \pm 7,2 \mathrm{~N})$. Tampouco houve diferença significante ( $\rho=0,434$, T-teste independente) entre essas referências aeróbias de carga.

O nível de correlação entre os parâmetros aeróbios (avaliados em nado atado e desimpedido), bem como destes com os desempenhos em nado desimpedido são apresentados na tabela 2. Os valores de $V_{\text {MFEL }}\left(1,17 \pm 0,11 \mathrm{~m} \cdot \mathrm{s}^{-1}\right)$ e $\mathrm{VPI}\left(1,19 \pm 0,11 \mathrm{~m} \cdot \mathrm{s}^{-1}\right)$ apresentam correlação elevada e positiva entre si e com $P_{\text {AtadamFeL }}$ ponderada pelo peso corporal $\left(1,29 \pm 0,24 \mathrm{~W} \mathrm{~kg}^{-1}\right)$. Assim como mostraram-se elevadas e positivas as correlações destes parâmetros aeróbios com o desempenho em $v_{400 m}\left(1,29 \pm 0,11 \mathrm{~m} \cdot \mathrm{s}^{-1}\right), \mathrm{v}_{800 \mathrm{~m}}\left(1,23 \pm 0,12 \mathrm{~m} \cdot \mathrm{s}^{-1}\right)$ e $\mathrm{v}_{1500 \mathrm{~m}}(1,21 \pm 0,12$ $\left.m . s^{-1}\right)$. Os valores das correlações apresentadas pelo parâmetro aeróbio em nado atado com os desempenhos em nado desimpedido (tabela 2) foram apenas ligeiramente menores do que aqueles apresentados pelos parâmetros aeróbios em nado desimpedido. Cabe destacar, que a intensidade em MFEL em nado atado e desimpedido associou-se a o limiar lactacidêmico de forma similar.

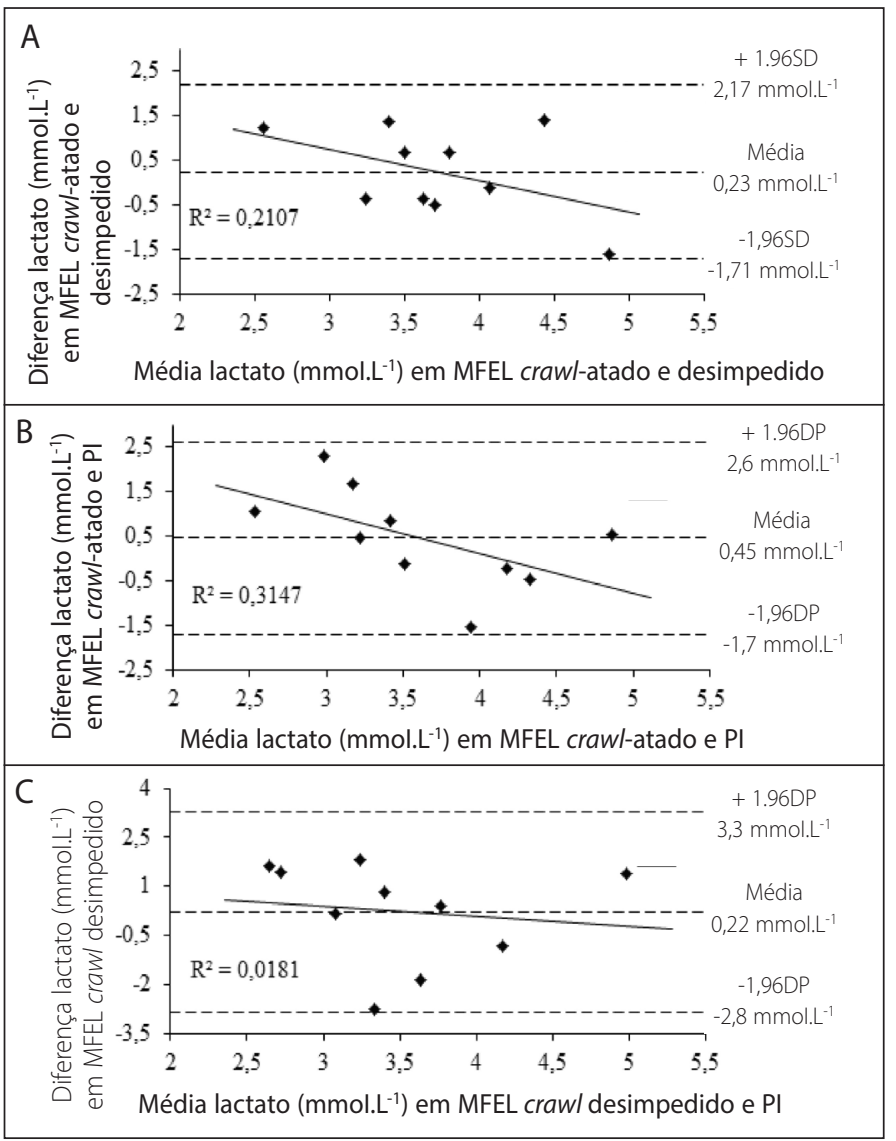

Figura 2. Concordância por Bland e Altman entre o perfil lactacidêmico em máxima fase estável de lactato (MFEL) no nado crawl atado e desimpedido (A); em MFEL no nado crawl atado e Ponto de Inflexão (PI) (B); e em MFEL no crawl desimpedido e PI (C).

Tabela 2. Coeficientes de Pearson entre os índices aeróbios e desempenho no nado crawl atado e desimpedido.

\begin{tabular}{|c|c|c|c|c|c|}
\hline & $\mathrm{v}_{400}\left(\mathrm{~m} \cdot \mathrm{s}^{-1}\right)$ & $\mathrm{v}_{800}\left(\mathrm{~m} \cdot \mathrm{s}^{-1}\right)$ & $\mathrm{v}_{1500}\left(\mathrm{~m} \cdot \mathrm{s}^{-1}\right)$ & $\mathrm{vPI}\left(\mathrm{m} \cdot \mathrm{s}^{-1}\right)$ & $\mathrm{v}_{\text {MFEL }}\left(\mathrm{m} \cdot \mathrm{s}^{-1}\right)$ \\
\hline$P_{\text {AtadaMFel }}\left(W \cdot \mathrm{kg}^{-1}\right)$ & $0,832^{* *}$ & $0,873^{* *}$ & $0,833^{* *}$ & $0,892^{* *}$ & $0,756^{* *}$ \\
\hline$v_{\text {MFEL }}\left(m \cdot s^{-1}\right)$ & $0,963^{* *}$ & $0.964^{* *}$ & $0,964^{* *}$ & $0,892^{* *}$ & - \\
\hline $\mathrm{vPI}\left(\mathrm{m} \cdot \mathrm{s}^{-1}\right)$ & $0,963^{* *}$ & $0,974^{* *}$ & $0,956^{* *}$ & --- & --- \\
\hline
\end{tabular}

$\mathrm{P}_{\text {AtadaMFEL }}$ - potência em máxima fase estável de lactato no nado atado; $V_{\text {MFEL }}$ - velocidade em máxima fase estáve de lactato; $\mathrm{VPI}$ - velocidade no ponto de inflexão. ${ }^{*} \mathrm{P}<0,01$.
A análise de dispersão entre os parâmetros aeróbios em nado atado e desimpedido com os desempenhos em nado desimpedido está apresentada na figura 3. A $P_{\text {AtadamFEL }}$ demonstra ter potencial entre moderado a elevado ( 70\%) em explicar as variações nos desempenhos de nado desimpedido, enquanto que $\mathrm{v}_{\text {MFEL }}$ e vPI apresentam elevado potencial explicativo ( 90-95\%) das variações nos desempenhos.

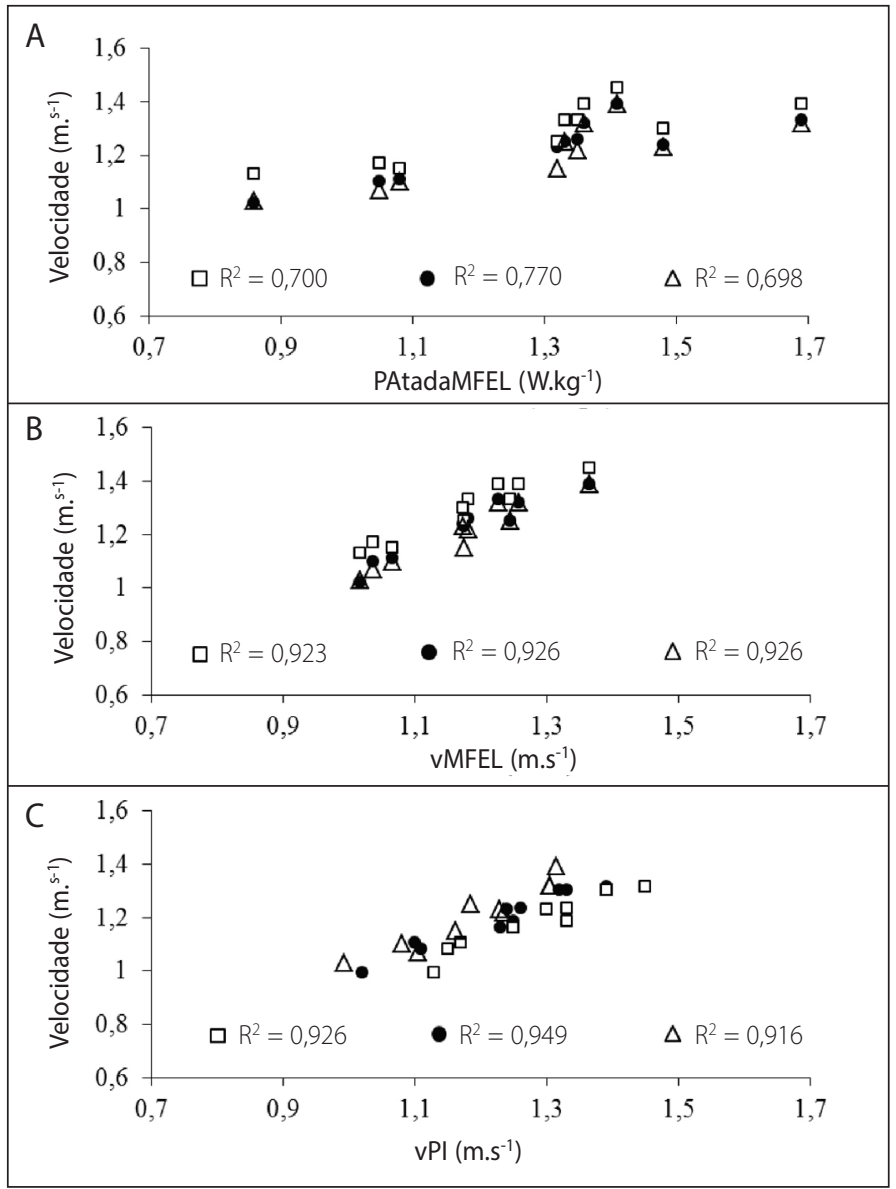

Figura 3. Análise de dispersão entre os parâmetros de potência em máxima fase estável de lactato (MFEL) no nado atado (A), velocidade em MFEL (B) e velocidade no ponto de inflexão (C) com os desempenhos em nado desimpedido: V400m (ם),

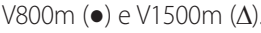

\section{DISCUSSÃO}

O presente estudo constatou a viabilidade de um teste simples de determinação da força de resistência ao nadar em velocidade máxima, como parâmetro individual de carga capaz de contextualizar diferentes zonas de exercício e graduar a intensidade do esforço em testes constantes no crawl atado. Assim como, o uso da assíntota do modelo carga-t $t_{\text {Lim }}$ por ajuste hiperbólico atendeu à expectativa de normalização de intensidades submáximas no crawl atado, com limites graduais e distintos do controle lactacidêmico, que atendem à precisão exigida na avaliação de MFEL.

Tem-se postulado que a concentração de lactato em 3 mmol...-1 seria um valor adequado para a MFEL na natação ${ }^{25}$. A observação direta da concentração correspondente à MFEL na natação apresenta valores médios de 2,8 \pm 1,2 $\mathrm{mmol}^{-\mathrm{L}^{-1}}$, correspondente a $88,4 \pm 2,9 \%$ da velocidade aeróbia máxima de nadadores entre 18-22 anos ${ }^{10}$. Estes valores são menores, mas na faixa de variação daqueles apresentados para a MFEL em nado atado $\left(3,84 \pm 0,60 \mathrm{mmol}^{-L^{-1}}\right)$ e desimpedido $\left(3,61 \pm 0,97 \mathrm{mmol}^{\left.\mathrm{L} \mathrm{L}^{-1}\right)}\right.$ no presente estudo. Também estão próximos aos valores reportados por Wakayoshi et al. ${ }^{18}$ que verificaram estabilidade na concentração de lactato em torno de 3,2 mmol..-1 durante esforços intermitentes de 400 metros desempenhados à 100\% daVC $\left(1,43 \pm 0,02 \mathrm{~m} \cdot \mathrm{s}^{-1}\right)$. 
Assim como outros estudos, que também analisaram a tendência de ocorrência de MFEL pela resposta fisiológica ao redor da VC, apresentando a concentração de lactato em 3,8 \pm 1,0 mmol. L $^{-1}$ como corresponden-

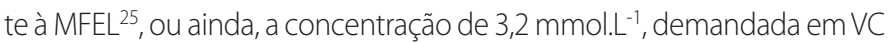
$\left(1,23 \pm 0,10 \mathrm{~m} \cdot \mathrm{s}^{-1}\right)$ estimada pelo modelo distância-tempo limite de ajuste linear, que representaria, segundo Martin e Whyte ${ }^{26}$, a intensidade capaz de induzir uma pequena acidose, mas que não causaria exaustão pela perturbação do equilíbrio ácido-base.

Filipatou et al. ${ }^{27}$ e Dekerle et al. ${ }^{12}$ observaram estabilidade da concentração de lactato somente em velocidade de nado abaixo de VC. Segundo esses autores, em velocidade $5 \%$ abaixo daquela correspondente às respectivas velocidades críticas $\left(1,34 \pm 0,04 \mathrm{~m} \cdot \mathrm{s}^{-1}\right.$ e 1,25 \pm 0,06 m.s $\left.\mathrm{s}^{-1}\right)$, os desempenhos intermitentes $(4 \times 400 \mathrm{~m})$ e constantes (até exaustão) demandaram variação entre $\left(3,8 \pm 0,9\right.$ mmol. $\left.\mathrm{L}^{-1}\right)$ a $\left(4,6 \pm 1,0 \mathrm{mmol} . \mathrm{L}^{-1}\right)^{27}$ e entre $\left(4,0 \pm 1,5 \mathrm{mmol} . \mathrm{L}^{-1} \text { a } 3,8 \pm 1,9 \mathrm{mmol}^{-1} \mathrm{~L}^{1}\right)^{12}$. No presente estudo, a referência de velocidade em MFEL $\left(1,17 \pm 0,11 \mathrm{~m} \cdot \mathrm{s}^{-1}\right)$ e o percentual $(90,7 \pm 2,2 \%)$ em relação à $v_{400 m}$ (considerada referência de velocidade de nado na potência aeróbia máxima - MVA ${ }^{10}$ ), mostram-se compatíveis à velocidade estimada para o ponto de inflexão da cinética lactacidêmica, bem como ao seu percentual $(92,4 \pm 2,4 \%)$ em relação à $v_{400 m}$.

A comparação entre a concentração de lactato em $v_{\text {MFEL }} e$ $P_{\text {AtadaMfEL }}$ não evidenciou diferenças significantes pela comparação entre as médias, tampouco houve discrepância entre os perfis lactacidêmicos (figura 2A). Além disto, houve similaridade na concordância entre estes índices de MFEL e o ponto de inflexão (figuras 2B e 2C), que é um tradicional método de avaliação da aptidão aeróbia por resposta lactacidêmica ${ }^{24}$. Também foi similar a magnitude e a forma como $V_{\text {MFEL }}$ e $P_{\text {Atadamfel }}$ correlacionaram-se aos desempenhos de média e longa distâncias no nado desimpedido (tabela 2 e figura 3). Para Beneke et al. ${ }^{1}$ e Beneke et al. ${ }^{1}$ a concentração de lactato respectiva à MFEL (obtida por protocolo de 30 minutos e com pontos de coletas ao 10e e 30 ำ minutos) contextualiza a intensidade do exercício, permitindo inferir sobre as similaridades nos padrões motores, na quantidade de massa muscular engajada na atividade, na demanda de potência por unidade de massa muscular, no nível de ativação da glicólise e na disponibilidade de $\mathrm{O}_{2}$ para a oxidação. Desta forma, pelos pressupostos envolvidos na determinação de MFEL, pode-se argumentar que a padronização de MFEL realizada em nado atado forneceu um parâmetro de carga de intensidade similar à modelagem de MFEL em nado desimpedido; e que não seria um equívoco considerar as intensidades em MFEL no nado atado e desimpedido proporcionais, quanto à qualidade motora e metabólica dos músculos engajados nas atividades.

Quanto à referência de potência aeróbia em nado atado, foram observados que os valores em MFEL $(51,7 \pm 5,6 \mathrm{~N}$ e 89,2 \pm 15,1 W, respectivamente), no presente estudo, não se mostram diferentes daqueles previstos pela equação da proporcionalidade mecânica $\left(P M F E L=\left(A^{*} V M F E L \wedge 3\right) / e p\right.$, proposta por Toussaint et al. $\left.{ }^{16}\right)$ para o nado desimpedido (94,8 $\pm 17,2 W$ W). Referências de potência no crawl desimpedido em intensidade que não leve ao acúmulo de lactato são escassas. Valores de potência, pela relação entre $\mathrm{VO}_{2}$ e a adição de cargas em uma piscina

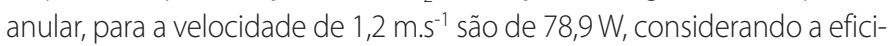
ência propulsiva equivalente a $51 \%$ no nado desimpedido e completo ${ }^{28}$. Shimonagata et al. ${ }^{29}$ verificaram um valor de $74,1 \mathrm{~W}$ correspondente à velocidade média de 1,29 m.s. ${ }^{-1}$, baseando-se em coeficientes de redução da velocidade e potência máximas em um teste de exaustão. Os valor médio de 115,4 \pm 18,4 W foi reportado por Swaine ${ }^{30}$ como correspondente ao limiar ventilatório (LV) e respectivo à $84,3 \pm 9,6 \% \mathrm{VO}_{2}$ pico obtido em um teste progressivo em banco de nado isocinético. Kolmogorov et al. ${ }^{31}$ previram valor médio de potência em 82,4 W para uma zona de intensidade de nado entre o Limiar de Lactato e o $\mathrm{VO}_{2 \max }$.
A potência em MFEL no crawl-atado, no presente estudo, apresentou correlações superiores àquelas que Ikuta et $a l .^{20}$ verificaram entre a força crítica no nado atado e $v_{400 m}(r=0,70)$, VC $(r=0,69)$ e com vOBLA $\left(r=0,68\right.$, por concentração fixa em 4 mmol. $\left.L^{-1}\right)$. Todavia, mostraram-se similares àquelas observadas por Papoti et al..$^{32}$ entre a força crítica e o limiar de lactato $(r=0,88)$, ambos avaliados em nado atado, e entre as velocidades nos 400m ( $r=0,85)$ e no teste de 30 minutos $(r=0,86)$. Assim como assemelham-se à correlação, reportada por Swaine ${ }^{33}$ entre PC em banco de nado $(120,7 \pm 9,4 \mathrm{~W})$ e o desempenho de nado em longa distância (1500 m, $r=-0,89)$.

Esses resultados apontam para a recomendação do nado atado em carga correspondente à MFEL como método específico para a avaliação aeróbia, com a vantagem de ser um ambiente controlado, além de permitir a análise das respostas ao treinamento pela acomodação da carga. No entanto, a paridade das adaptações aeróbias (centrais e periféricas) obtidas com o treinamento em MFEL no nado atado, diante daquelas propiciadas em nado desimpedido, precisam ser alvos de pesquisas futuras que sistematizem o exercício em ambas as condições e as comparem quanto à especificidade e transferência das adaptações ao desempenho de nado.

Essa é uma limitação do presente estudo, porque a diferença entre as condições de nado está na mecânica gestual e no seu controle ${ }^{15}$, que possivelmente comprometem uma análise conclusiva das respostas fisiológicas ao nado atado em MFEL. Se, por um lado, as respostas fisiológicas demonstram não haver vantagem do ambiente em nado atado por ser capaz de reduzir as variações na velocidade e força do hidrofólio durante a braçada, resultando na habilidade de evitar o desperdício de potência ${ }^{34,35}$; por outro lado, particularidades do ciclo gestual em nado atado sugerem deteriorização de sua mecânica (redução do comprimento de braçada) em comparação ao nado desimpedido, que reduz sua economia com a progressão da intensidade ${ }^{28}$.

Outra limitação seria o método empregado para determinação da referência de força máxima. A aplicação da Eq. 1 fornece uma aproximação do equilíbrio entre as forças de propulsão e arrasto ativo, ao considerar um princípio básico: a associação da força de arrasto ativo com o quadrado da velocidade nado ${ }^{16,36}$. Contudo, sua utilização reúne praticidade do protocolo, facilidade de interpretação e baixo custo operacional ao pensar no dia-a-dia do profissional à beira da piscina. Um procedimento com validade e reprodutibilidade de medida da força atada é a célula de carga acoplada ao nadador por um cabo inelástico. Este procedimento é reconhecidamente uma medida da força de propulsão máxima ${ }^{34,37}$ e correlacionada ao desempenho do crawl em nadadores jovens ${ }^{36,38}$ e adultos $^{39}$. No entanto, os valores fornecidos pela Equação 1, no presente estudo, mostram-se compatíveis aos valores registrados para homens $\left(\mathrm{Fr}=30 \mathrm{v}^{2}\right)$ e mulheres $\left(\mathrm{Fr}=24 \mathrm{v}^{2}\right)$ correspondentes às velocidades de 1,86 e 1,63 m.s. $\mathrm{s}^{-1}$, respectivamente, assim como a referência geral da força de arrasto ( $F r$ 21,33v 2.34$)^{37}$, em uma comparação entre os principais métodos de determinação da força de arrasto. Estas semelhanças asseguram a validade do procedimento adotado para determinar a intensidade do nado nas avaliações em condições atada.

\section{CONCLUSÃO}

Conclui-se que a aplicação de MFEL em nado atado é válida para acessar o desempenho aeróbio, por suas relações com o desempenho e com as respostas fisiológicas apresentadas por outros índices da aptidão aeróbia de nado. Enquanto recurso de treinamento, a intensidade de nado atado demonstra ser promissora, dada às correlações que apresenta com o desempenho em distâncias médias e longas na natação.

Todos os autores declararam não haver qualquer potencial conflito de interesses referente a este artigo. 


\section{REFERÊNCIAS}

1. Beneke R, Leithäuser RM, Hütler M. Dependence of the maximal lactate steady-state on the motor pattern of exercise. Br J Sports Med. 2001;35(3):192-6.

2. Beneke R. Maximal lactate steady state concentration (MLSS): experimental and modelling approaches. Eur J Appl Physiol. 2003;88(4-5):361-9.

3. Billat VL, Sirvent PYG, Koralsztein J-P, Mercier J. The concept of maximal lactate steady state a bridge between biochemistry, physiology and sport science. Sports Med. 2003;33(6):407-6.

4. Dekerle J, Baron B, Dupont L, Vanvelcenaher J, Pelayo P. Maximal lactate steady state, respiratory compensation threshold and critical power. Eur J Appl Physiol. 2003;89(3-4):281-8.

5. Jones AM, Vanhatalo A, Burnley M, Morton RH, Poole DC. Critical power: implications for determination of V'O2max and exercise tolerance. Med Sci Sports Exerc. 2010;42(10):1876-90.

6. Smith C, Jones A. The relationship between critical velocity, maximal lactate steady-state velocity and lactate turnpoint velocity in runners. Eur J Appl Physiol. 2001;85(1):19-26.

7. Kjendlie P-L, Stallman R. The validity of a non-paced lactate profile test for swimmers. Rev Port Ciênc Desp. 2006;6(Suppl 2):140-2.

8. Ungerechts $B$. The relation of peak body acceleration to phases of movements in swimming. In: Ungerechts B, Wilke K, Reischle K, editors. Swimming science V. Champaign, llinois: Human Kinetics; 1988. p. 61-6.

9. Nomura T, Shimoyama Y. The relationship between stroke parameters and physiological resposnses at the various swim speeds. In: Chatard JC. Biomechanics and Medicine in Swimming IX. Saint-Etienne: Université de Saint-Etienne; 2003. p. 355-60.

10. Dekerle J, Pelayo P, Clipet B, Depretz G, Lefevre T, Sidney M. Critical swimming speed does not represent the speed at maximal lactate steady state. Int J Sport Med. 2005;26(7):524-30.

11. Kjendlie PL, Thorsvald K. A tethered swimming power test is high reliable. Rev Port Ciênc Desp. 2006;6(Suppl 2):231-5.

12. Dekerle J, Brickley G, Alberty M, Pelayo P. Characterinzing the slope of the distance-time relationship in swimming. J Sci Med Sport. 2010;13(3):365-70.

13. Dekerle J. The use of critical velocity in swimming: a place for critical stroke rate? Biomechanics and Medicine in Swimming X. Rev Port Ciênc Desp. 2006;6(Suppl 2):201-5.

14. Pessôa Filho DM, Nascimento EP, Denadai BS. Validação do modelo potência-tempo limite no crawl-atado pelas relações com os modelos distância/tempo limite e velocidade/tempo limite. Rev Bras Ciênc Esp. 2008;29(2):95-114.

15. Pessôa Filho DM, Denadai BS. Mathematical basis for modeling swimmer power output in the front crawl tethered swimming: an application to aerobic evaluation. The Open Sports Sci J. 2008;7(1):31-7.

16. Toussaint H, Wakayoshi K, Hollander A, Ogita F. Simulated front crawl swimming performance related to critical speed and critical power. Med Sci Sports Exerc. 1998;30(1):144-51.

17. Bishop D, Jenkins DG, Howard A. The critical power function is dependent on the duration of the predictive exercise tests chosen. Int J Sports Med. 1998;19(2):125-9.

18. Wakayoshi K, Yoshida T, Udo M, Harada T, Moritani T, Mutoh Y, et al. Does critical swimming velocity represent exercise intensity at maximal lactate steady state? Eur J Appl Physiol. 1993;66(1):90-5.

19. Zacca R, Wenzel BM, Piccin JS, Marcilio NR, Lopes AL, de Souza Castro FA. Critical velocity, anaerobic distance capacity, maximal instantaneous velocity and aerobic inertia in sprint and endurance young swimmers. Eur J Appl Physiol. 2010;110(1):121-31

20. Ikuta Y, Wakayoshi K, Nomura T. Determinations and validity of critical swimming force as performance index in tethered swimming. In: Troup JP, Hollander AP, Strasse D, Trappe SW, Cappaert JM, Trappe TA, editors. Biomechanics and Medicine in Swimming VII. London: E \& FN SPON; 1996. p. 146-51.
21. Pessôa Filho DM, Denadai BS. Extending the critical force model to approach critical power in tethered swimming, and its relationship to the indices at maximal lactate steady-state In: XIth International Symposium for Biomechanics and Medicine in Swimming XI ed. Oslo, Noruega : Nordbergtrykk, 2010.

22. Schleihauf RE, Gray L, DeRose J. Three dimensional analysis of hand propulsion in the sprint front crawl stroke. In: Hollander A, Huijing P, DeGroot G, editors. Biomechanics and medicine in swimming III. Champaign, Ilinois: Human Kinetics; 1983. p. 184-91.

23. Beaver WL, Wasserman K, Whipp BJ. A new method for detecting threshold by gas exchange. J Appl Physiol. 1986;60(6):2020-7.

24. Bentley DJ, Newell J, Bishop D. Incremental exercise test design and analysis: implications for performance diagnostics in endurance athletes. Sports Med. 2007;37(7):575-86.

25. Takahashi S, Wakayoshi K, Hayashi A, Sakaguchi Y, Kitagawa K. A method for determining critical swimming velocity. Int J Sports Med. 2009;30(2):119-23.

26. Martin L, Whyte GP. Comparison of critical swimming velocity and velocity at lactate threshold in elite triathletes. Int J Sports Med. 2000;21(5):366-8.

27. Filipatou E, Toubekis A, Douda H, Pilianidis T, Tokmakidis S. Lactate and heart rate responses during swimming at $95 \%$ and $100 \%$ of the critical velocity in children and young swimmers. Rev Port Ciênc Desp. 2006;6(Suppl 2):132-4.

28. Zamparo P, Bonifazi E, Faina M, Milan A, Sardella F, Schena F, et al. Energy cost of swimming of elite long-distance swimmers. Eur J Appl Physiol. 2005;94(5-6):697-704.

29. Shimonagata S, Taguchi M, Miura M. Effect of swimming power, swimming power endurance and dry-land power on 100m freestyle performance. In: Chatard JC. Biomechanics and medicine in swimming IX. Saint-Etienne: University of Saint Etienn; 2003. p. 391-6.

30. Swaine IL. The relationship between physiological variables from a swim bench ramp test and midlledistance swimming performance. J Swim Res. 1994;10(1):41-8.

31. Kolmogrov SV, Vorontsov AR, Rumyantseva OA, Kochergin AB. Mechanical and propulsive efficiency of swimmers in diffrent zones of energy supply. In: Kjendlie PL, Stallman RK, Cabri J. editors. Biomechanics and medicine in swimming XI. Oslo: Norwegian School of Sport Science; 2010. p. 110-2.

32. Papoti M, Vitório R, Araújo GS, Martins LEB, Cunha AS, Gobatto, CA. Força crítica em nado atado para avaliação da capacidade aeróbia e predição de performances em nado livre. Rev Bras Cineantropom Desempenho Hum. 2010;12:14-20.

33. Swaine IL. The relationship between $1500 \mathrm{~m}$ swimming performance and critical Power using an isokinetic swim bench. In: Troup JP, Hollander AP, Strasse D, Trappe SW, Cappaert JM, Trappe TA. Biomechanics and medicine in swimming VII. London: E \& FN SPON; 1996. p. 229-33.

34. Rouard AH, Aujouannet YA, Hintz F, Bonifazi M. Isometric force, tethered force and power ratios as tools for the evaluation of technical ability in freestyle swimming Rev Port Ciênc Desp. 2006.6(Suppl 2):249-50.

35. Vorontsov A, Popov O, Binevsky D, Dyrko V. The assessment of specifcis strength in well-trained male athletes during tethered swimming in the swimming flume. Rev Port Ciênc Desp. 2006;6(Suppl 2):275-7.

36. Toussaint HM, Roos PE, Kolmogorov S. The determination of drag in front crawl swimming. J Biomech. 2004;37(11):1655-63.

37. Morouço P, Keskinen KL, Vilas-Boas JP, Fernandes RJ. Relationship between tethered forces and the four swimming techniques performance. J Appl Biomech. 2011;27(2):161-9.

38. Ria B, Falgairette G, Robert A. Assessment of the mechanical Power in the young swimmer. J Swim Res. 1990;6(1):11-5.

39. Johnson R, Sharp R, Hedrick C. Relationship of swimming power and dryland power to sprint freestyle performance: a multiple regression approach. J Swim Res. 1993;9(1):10-4. 